\title{
A RESIDUE MAP AND ITS APPLICATIONS TO SOME ONE-DIMENSIONAL RINGS
}

\author{
I-CHIAU HUANG
}

(Communicated by Wolmer V. Vasconcelos)

\begin{abstract}
A residue map is used to study canonical modules of the ring $k\left[\left[X^{t_{1}}, \ldots, X^{t_{n}}\right]\right]$. A simple proof of a well-known numerical criterion for $k\left[\left[X^{t_{1}}, \ldots, X^{t_{n}}\right]\right]$ to be Gorenstein is given.
\end{abstract}

Let $k$ be a field and $R$ be the one-dimensional local ring $k\left[\left[X^{t_{1}}, \ldots, X^{t_{n}}\right]\right]$, where $\left(i_{1}, \ldots, t_{n}\right)=1$. Denote by $\mathfrak{m}$ the maximal ideal of $R$. It is well known that injective hulls of an $R$-module are isomorphic to each other. However, finding isomorphisms between them is a very subtle question. For example, the $R$-module $\operatorname{Hom}_{k}^{c}(R, k)$ consisting of all $R$-linear maps $u: R \rightarrow k$, which are continuous for the m-adic topology of $R$ and the discrete topology of $k$ (i.e., $u\left(\mathfrak{m}^{n}\right)=0$ for some $n$ ), is an injective hull of $R / \mathfrak{m}$ [3, Proposition 3.4]. Another injective hull of $R / \mathfrak{m}$ is given by local cohomology: Let $k\left[\left[X_{1}, \ldots, X_{n}\right]\right] \rightarrow R$ be the canonical map sending $X_{i}$ to $X^{t_{i}}$. $\operatorname{Hom}_{k\left[\left[X_{1}, \ldots, X_{n}\right]\right]}\left(R, H_{X_{1}, \ldots, X_{n}}^{n}\left(k\left[\left[X_{1}, \ldots, X_{n}\right]\right]\right)\right)$ is also an injective hull of $R / \mathfrak{m}$ [3, Propositions 3.4 and 3.8]. An isomorphism

$$
\operatorname{Hom}_{k\left[\left[X_{1}, \ldots, X_{n}\right]\right]}\left(R, H_{X_{1}, \ldots, X_{n}}^{n}\left(k\left[\left[X_{1}, \ldots, X_{n}\right]\right]\right)\right) \simeq \operatorname{Hom}_{k}^{c}(R, k)
$$

can be described using a residue map (cf. [3]).

A similar phenomenon occurs in canonical modules. Denote by $(-)^{\wedge}$ the functor $\operatorname{Hom}_{R}\left(-, \operatorname{Hom}_{k}^{c}(R, k)\right)$. A finitely generated $R$-module $K$ is called a canonical module if there is a functorial isomorphism

$$
\left(\operatorname{Ext}^{1-i}(M, K)\right)^{\wedge} \simeq H_{\mathfrak{m}}^{i}(M)
$$

for finitely generated $R$-module $M(i=0,1)$. It is easy to see that any canonical module, if it exists, is isomorphic to $\left(H_{\mathfrak{m}}^{1}(R)\right)^{\wedge}$. But in general isomorphisms between canonical modules are not easy to describe. This note gives an explicit description of a canonical module $K$ as a submodule of $k[[X]]$ and describes explicitly an isomorphism from $K$ to $\left(H_{\mathrm{m}}^{1}(R)\right)^{\wedge}$ using a residue map. The main purpose of this note is to bring out some concrete aspects of Grothendieck's duality theory into the open, so that the structure of $R$ is better understood.

Received by the editors October 15, 1993 and, in revised form, December 16, 1993.

1991 Mathematics Subject Classification. Primary 13J05; Secondary 13H10.

Key words and phrases. Canonical module, dualizing complex, Gorenstein ring, residue. 
This note uses freely basic properties of Matlis duality, which is referred to [6].

Let $\alpha$ be the conductor of $R$ (i.e., the smallest number such that $X^{i} \in R$ for all $i \geq \alpha)$. The residue map we need in this note is the $k$-linear map

$$
\text { res: } k((X)) \rightarrow k
$$

defined by $\operatorname{res}\left(\sum a_{i} X^{i}\right)=a_{\alpha-1}$ for $\sum a_{i} X^{i}$ in $k((X))$. It induces an $R$ isomorphism from $k((X))$ to its dual.

Lemma 1. The R-linear map

$$
\Phi: k((X)) \rightarrow k((X))^{\wedge},
$$

defined by $\Phi(f)(g)(r)=\operatorname{res}(f g r)$ for $f, g \in k((X))$ and $r \in R$, is an isomorphism.

Proof. Let $f=a_{-m} X^{-m}+\cdots+a_{0}+a_{1} X+\cdots$ be an element in $k((X))$. Since $\Phi(f)\left(X^{\alpha+m-1}\right)(1)=a_{-m}, \Phi(f)=0$ implies $f=0$. Given $\phi \in k((X))^{\wedge}$ and $X^{j} \in R$,

$$
\phi\left(X^{i}\right)\left(X^{j}\right)=\left(X^{j} \phi\left(X^{i}\right)\right)(1)=\phi\left(X^{i+j}\right)(1) .
$$

Hence $\phi\left(X^{l}\right)$ vanishes for $l$ sufficiently large. Define

$$
f:=\sum_{i \in \mathbb{Z}} \phi\left(X^{i}\right)(1) X^{\alpha-1-i} .
$$

Then $\Phi(f)\left(X^{l}\right)(1)=\phi\left(X^{l}\right)(1)$. Since $\phi$ is determined by $\phi\left(X^{l}\right)(1)$, it follows that $\Phi(f)=\phi$.

Proposition 2. The map $\Psi: k((X)) \rightarrow \operatorname{Hom}_{k}^{c}(R, k)$ sending $f \in k((X))$ to the map $r \mapsto \operatorname{res}(f r)$ is $R$-linear and surjective.

Proof. The proposition can be verified easily and directly. It also follows from the fact that $\Psi$ is the composition

$$
k((X)) \stackrel{\Phi}{\rightarrow} k((X))^{\wedge} \rightarrow \operatorname{Hom}_{k}^{c}(R, k),
$$

where the second map $k((X))^{\wedge} \rightarrow \operatorname{Hom}_{k}^{c}(R, k)$ is the dual of the inclusion map $R \rightarrow k((X))$.

We remark that

$$
k((X)) \stackrel{\Psi}{\rightarrow} \operatorname{Hom}_{k}^{c}(R, k)
$$

is actually a dualizing complex of $R$. The proof is left to the reader who is familiar with dualizing complexes. The kernel $K$ of the map $\Psi$ has a simple description: Let $S$ be the semigroup generated by $t_{1}, \ldots, t_{n}$. Denote by $S^{\wedge}$ the subset of $\mathbb{Z}$ consisting of those elements $i$ such that $\alpha-1-i \notin S$. Then

$$
K=\left\{\sum a_{i} X^{i} \mid a_{i} \in k \text { and } i \in S^{\wedge}\right\} .
$$

Since $S^{\wedge}$ is a subset of $\mathbb{N}, K$ is an $R$-submodule of $k[[X]]$.

Recall that $R$ is Gorenstein if it has finite injective dimension. A necessary and sufficient condition for $R$ to be Gorenstein is that it has an injective resolution

$$
0 \rightarrow R \stackrel{\text { inclusion }}{\longrightarrow} k((X)) \rightarrow \operatorname{Hom}_{k}^{c}(R, k) \rightarrow 0
$$

for some $R$-linear map $k((X)) \rightarrow \operatorname{Hom}_{k}^{c}(R, k)$ [6, Theorem 18.8]. As a consequence of the proposition and the fact that $k((X))$ and $\operatorname{Hom}_{k}^{c}(R, k)$ are injective $R$-modules, we get a simple proof of the following theorem due to Kunz [5]. 
Corollary 3. If $S$ is a symmetric (i.e., $S=S^{\wedge}$ ), $R$ is Gorenstein.

Theorem 4. $K$ is a canonical module of $R .^{1}$

Proof. $K$ is finitely generated because it is generated by $X^{i}, i \in S^{\wedge}$ and $i<2 \alpha$. Let $M$ be a finitely generated $R$-module. $\left(\operatorname{Ext}^{1-i}(M, K)\right)^{\wedge}$ is the $i$ th homology of the following complex induced by $\Psi$ :

$$
M^{\wedge \wedge} \stackrel{d}{\longrightarrow} \operatorname{Hom}_{R}(M, k((X)))^{\wedge} .
$$

By Matlis duality, the canonical map $M \rightarrow M^{\wedge \wedge}$ is an isomorphism. We claim that the map

$$
\Phi_{M}: M_{X^{\alpha}} \rightarrow \operatorname{Hom}_{R}(M, k((X)))^{\wedge}
$$

defined by $\Phi_{M}\left(\frac{m}{X^{l \alpha}}\right)(\phi)(r)=\operatorname{res} \frac{r \phi(m)}{X^{l \alpha}}$ for $\phi \in \operatorname{Hom}_{R}(M, k((X)))$ and $r \in R$ is an $R$-isomorphism. Since the functors $(-)_{X^{\alpha}}$ and $\operatorname{Hom}_{R}(-, k((X)))^{\wedge}$ are additive and exact, and $M$ is finitely generated, to prove the claim we may assume that $M=R$. In such case $R_{X^{\alpha}}=k((X))$, so the claim follows from Lemma 1. The theorem follows form the observation that the diagram

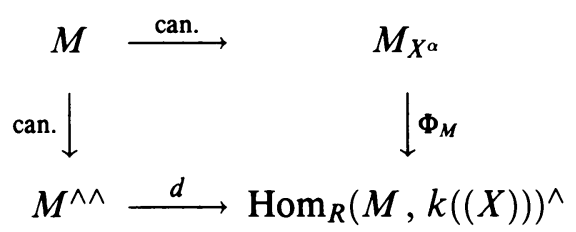

is commutative.

We have a simple proof of the following result which is contained in [1] and [4].

Corollary 5. Some ideals of $R$ are canonical modules.

Proof. The map $K \rightarrow k((X))$, multiplied by $X^{\alpha}$, is $R$-linear and injective with image in $R$.

The converse of Corollary 3, also due to Kunz [5], has a simple proof.

Theorem 6. If $R$ is Gorenstein, $S$ is symmetric.

Proof. $R$ has an injective resolution

$$
0 \rightarrow R \stackrel{\text { inclusion }}{\longrightarrow} k((X)) \rightarrow \operatorname{Hom}_{k}^{c}(R, k) \rightarrow 0
$$

with some unknown map $k((X)) \rightarrow \operatorname{Hom}_{k}^{c}(R, k)$. Taking dual, then applying Matlis duality and Lemma 1, we get an exact sequence:

$$
0 \rightarrow R \rightarrow k((X)) \stackrel{\Psi}{\longrightarrow} \operatorname{Hom}_{k}^{c}(R, k) \rightarrow 0 .
$$

This implies $K \simeq R$, although the map $R \rightarrow k((X))$ above is still unknown. Since $1 \in K, K$ is generated by a power series $f$ whose constant coefficient is 1 and there exists

$$
g=1+\sum_{i>0, i \in S} a_{i} X^{i}
$$

\footnotetext{
'Professor Herzog informed me that this description of canonical module is implicitly in [2].
} 
in $R$, such that $g f=1 . S$ is a semigroup, hence

$$
f=1-\left(\sum a_{i} X^{i}\right)+\left(\sum a_{i} X^{i}\right)^{2}-\left(\sum a_{i} X^{i}\right)^{3}+\cdots \in R .
$$

Therefore $K=R$ (as subsets of $k[[X]]$ ). Equivalently $S=S^{\wedge}$.

$H_{\mathrm{m}}^{1}(R)$ is isomorphic to the cokernel of the map

$$
R \stackrel{\text { inclusion }}{\longrightarrow} R_{X^{\alpha}} \text {. }
$$

Since $R_{X^{\alpha}}=k((X)), H_{\mathfrak{m}}^{1}(R) \simeq k((X)) / R$. The image of $K$ of the map

$$
\Phi: k((X)) \rightarrow k((X))^{\wedge}
$$

consists of all $\phi \in k((X))^{\wedge}$ with $\phi(R)=0$. Therefore we get an isomorphism

$$
K \stackrel{\sim}{\rightarrow}(k((X)) / R)^{\wedge} \text {. }
$$

Example 7. $R=k\left[\left[X^{7}, X^{9}, X^{10}\right]\right]$.

$S=\{0,7,9,10,14,16,17,18,19,20,21,23,24,25,26,27,28$, $29, \ldots\}$.

The conductor $\alpha=23$.

$S^{\wedge}=\{0,7,9,10,11,14,16,17,18,19,20,21,23, \ldots\}$.

$X^{7} K=R X^{7}+R X^{18}$ is a canonical module of $R$.

\section{ACKNOWLEDGMENT}

Part of this work was done while the author was visiting Purdue University. The author would like to thank the Department of Mathematics, Purdue University for hospitality and the Institute of Mathematics, Academia Sinica for financial support.

\section{REFERENCES}

1. Herzog and E. Kunz, eds., Der Kanonische Modul eines Cohen-Macaulay-Rings, Lecture Notes in Math., vol. 238, Springer-Verlag, Berlin and New York, 1971.

2. J. Herzog and E. Kunz, Die Werthalbgruppe eines lokalen Rings der dimension 1, Sitzungsber. Heider. Akad. Wiss., Springer-Verlag, Berlin and New York, 1971.

3. I-C. Huang, Pseudofunctors on modules with zero dimensional support, Mem. Amer. Math. Soc. (to appear).

4. J. Jäger, Längenberechnung und kanonische ideale in eindimensionalen ringen, Arch. Math. 29 (1977), 504-512.

5. E. Kunz, The value semigroup of a one-dimensional Gorenstien ring, Proc. Amer. Math. Soc. 25 (1970), 748-751.

6. H. Matsumura, Commutative ring theory, Cambridge Univ. Press, Cambridge and New York, 1986.

Institute of Mathematics, Academia Sinica, Nankang, Taipei 11529, Taiwan, Republic OF CHINA

E-mail address: maichiau@ccvax.sinica.edu.tw 